\title{
Correlation of Objective Assessment of Facial Paralysis with House-Brackmann Score
}

\author{
Wan Syahirah W Samsudin ${ }^{\star 1}$, Rosdiyana Samad ${ }^{2}$, Kenneth Sundaraj $^{3}$, Mahfuzah Mustafa $^{4}$, \\ Nor Rul Hasma Abdullah ${ }^{5}$ \\ 1,2,4,5 Faculty of Electrical \& Electronic Engineering, Universiti Malaysia Pahang, Malaysia \\ ${ }^{3}$ Faculty of Electronics \& Computer Engineering, Universiti Teknikal Malaysia Melaka, Malaysia \\ ${ }^{*}$ Corresponding author, e-mail: wansyahirahwsamsudin@yahoo.com
}

\begin{abstract}
This article illustrated a brief review of some objective methods in assessing facial nerve function for facial nerve paralysis which were correlated with House-Brackmann Grading System (HBGS). A rigorous search of online databases such as Springer, Elsevier and IEEE was conducted from June, 2015 to November, 2016 to discover and analyze the previous works in facial nerve assessment methods for facial paralysis. Several domains such as facial grading system and methods used to evaluate the facial nerve function were extracted for further analysis. Different keywords were used to acquire the studies based on the desire criteria. A total of 8 articles were identified and were analyzed for inclusion in this search. In conclusion, this review has presented an initial overview for further improvements in objective facial nerve assessment which has to be correlated with subjective assessment to make it more reliable and useful in clinical practice.
\end{abstract}

Keywords: facial nerve assessment, facial nerve paralysis, house-brackmann, facial grading system, facial rehabilitation

Copyright $@ 2017$ Universitas Ahmad Dahlan. All rights reserved.

\section{Introduction}

The facial nerve is seventh cranial nerve where the damage of any this nerve can cause facial nerve paralysis. Patient will not be able to move some or all of the facial muscles on one side of the face when the paralysis occurs. Numerous conditions can cause facial nerve paralysis including infections, inherited diseases, tumors, toxins, and trauma. Bell's palsy is $50 \%$ of the causes and it comes without identifiable cause [1,2].

Nowadays, facial paralysis continues as critical issue in clinical regardless of developed and sophisticated medical [3] and physical therapy interventions. A great numbers of measurement scales of facial nerve function have been proposed in the literature over the years. In 1983, House has proposed a gross facial score [4] and in 1985, it was modified to House-Brackmann Grading System (HBGS) as shown in Table 1. This latter system [5] has been adopted as the North America Standard in reporting results of facial nerve paralysis. The HBGS is very helpful in assessment of facial nerve function and although many of the other grading scales have their own advantages, none has duplicated the global appeal and ease of use of the HBGS [6]. However, it has been criticized for being not having sufficient in sensitivity and not effective for determining changes in facial nerve function following a therapeutic intervention [7]. From these aspects, several current works have proposed objective facial nerve assessment methods in order to overcome the limitation of HBGS. Yet, they still use the HBGS as their reference in presenting the results or accuracy of their proposed methods.

The aim of this review is to present a brief overview of objective facial grading systems or facial nerve assessment methods which used HBGS as ground truth to their studies because the proposed objective methods should be compared with the current subjective standards in clinical use to complement each other in facial nerve paralysis cases [7] and HBGS is found to be more fast and reliable for clinical practice [8]. 


\section{Research Method}

A thorough search of articles was conducted using online databases such as IEEE, Wiley online library, PubMed, Elsevier, Springer, and the ACM digital from June, 2015 to November, 2016 to discover and analyze the previous works in facial nerve assessment method for facial paralysis. The search has begun with some keywords pertaining to facial nerve paralysis, facial nerve assessment method, facial grading system, Bell's palsy assessment, and the others combined with the medical terms in order to distinguish the definition, causes and symptoms, reported cases, and any knowledge related to facial nerve paralysis. In avoiding any selection bias, several inclusion and exclusion criteria have been set before the data extraction and analysis as shown in Figure 1.

A total of 150 articles were found during the search period based on title, keywords, abstract and these appeared to be qualified for this review at early stage. The titles were then examined for potential relevance. All of these studies were considered based on the following desired criteria: (a) published in the English language; (b) objective facial nerve assessment methods; and (c) used House-Brackmann (HB) score as the reference. A total of 8 studies were included and analyzed in this review after the searched process. The researchers will be able to get a brief review of facial nerve evaluation methods and can compare the other subjective scale than House-Brackmann (HB) to be considered for correlating the final results of their study in order to develop an efficient and reliable assessment method for facial nerve function.

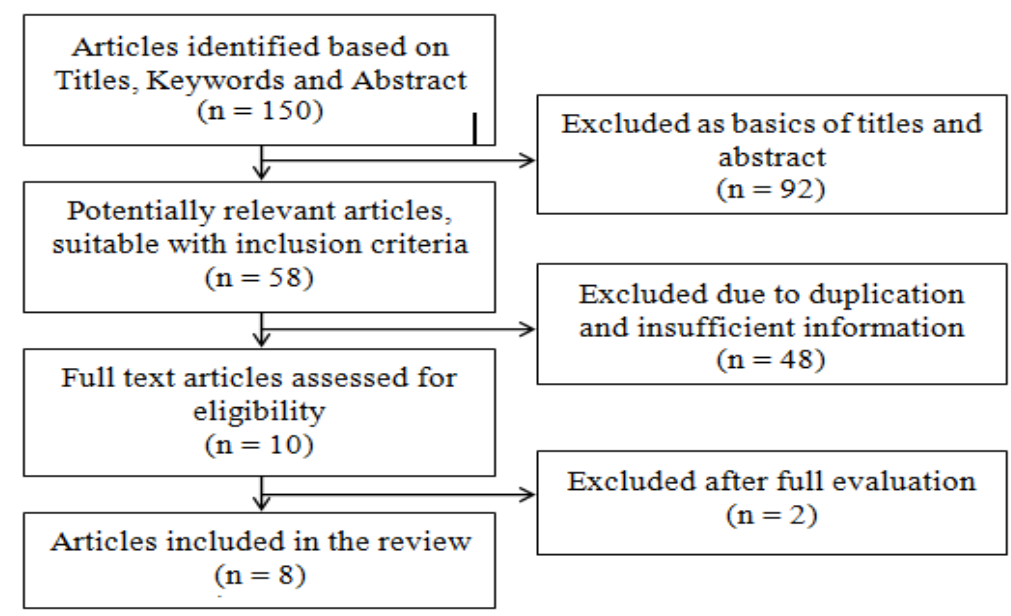

Figure 1. Illustration of search strategy

\section{Results and Analysis}

In this section, it is explained the results of research and at the same time is given the comprehensive discussion. Results can be presented in figures, graphs, tables and others that make the reader understand easily $[2,5]$. The discussion can be made in several sub-chapters.

\subsection{The House-Brackmann Grading System (HBGS)}

The House-Brackmann Grading System (HBGS) is a widely used scale and until now, it is still considered as universal standard all over the world. The HBGS is differed from the traditional HB gross score since this modified system provided regional assessment for the forehead, eye, nose and mouth. Yen et al [9] have proved by using regional assessment of HBGS, it was more fully efficient to analyze the communication of facial nerve functions. The system has assessed four regions of face and assigned a score of 1 to 6 for the degree of movement. A scale of 0 to 3 is scored for the synkinesis problem over the face. The addition of these two scores gave out a final score of 4 to 24 , which then converted to a grade of HBGS. Even though this system is taking longer to perform, however it is more convenient compared to the traditional House-Brackmann Scale. 


\subsection{How the Objective Methods Correlated with HBGS}

The measurement of HBGS is determined by measuring upwards movement of midportion of top of eyebrow and outwards movement of angle of mouth. Wang et al [10] have proposed a similar method with the HBGS system. They have focused on facial parts which involved for each facial movement. For example, the change in region of the eyebrows and eyes are examined by clinician during the raising eyebrows movement. In this work, the facial asymmetry features from combined regions for each facial movement as shown in Table 1 were extracted.

Table 1. Fused regions for each facial movement

\begin{tabular}{cc}
\hline Facial Movement & Fused Regions \\
\hline Raise Eyebrows & Eye \& Eyebrows \\
Close Eyes & Eyebrow, Eye \& Upper Nose \\
Screw-up Nose & Eye, Upper Nose \& Lower Nose \\
Plump Cheek & Lower Nose \& Cheek \\
Open Mouth & Lower Nose, Cheek \& Mouth \\
\hline
\end{tabular}

For the system validation, ten professional doctors are invited and have been asked to grade using percentage to standardize the grade representation. The relationship between the doctors' grade and the HBGS is illustrated in Table 2. Then, from all the scores graded by ten doctors were averaged and used as the ground truth.

Table 2. The relationship between doctors' scores and House-Brackmann grading system

\begin{tabular}{cc}
\hline Doctors' grading standard & House-Brackmann Grading System \\
\hline $0 \leq x \leq 10$ & I \\
$10<x \leq 30$ & II \\
$30<x \leq 50$ & III \\
$50<x \leq 70$ & IV \\
$70<x \leq 90$ & V \\
$90<x \leq 100$ & VI \\
\hline
\end{tabular}

In order to obtain the facial asymmetry features, they have proposed a method which combined both the static and dynamic factor. The facial asymmetry at maximal movement was evaluated as static and for the dynamic; the changes of muscles during facial movement were evaluated before speed of the changes were calculated for each different regions. They used Active Shape Model (ASM) in locating 68 key points on images and Local Binary Pattern (LBP) features were extracted in representing the facial asymmetry on the left and right side of face. This proposed method is claimed to be suitable in evaluating the degrees of facial paralysis of unlabeled image or disordered video data.

In other work, Jane and Thomas [11] have implemented Active Appearance Models (AAM) in extracting information related to the shape and location of facial features which are required for HBGS. Two important measurements in HBGS are upwards movement of the eyebrows and outwards movement of the angle of mouth. Their implementation is to measure the ability of patient to smile. In this study, a facial dataset consists of synthesized image of various level of paralysis has been developed. The distance between the corners of the mouth and those on each of subsequent image were measured. By using the HBGS, the value of distance which specifically related to the outwards movement of the corners of mouth was interpreted as illustrated in Table 3. 
Table 3. Implementation of Modified HBGS

\begin{tabular}{cll}
\hline Grade & Description & Measurement \\
\hline I & Normal & $d>1.0$ \\
II & Slight & $1.0<d>0.75$ \\
III & Moderate & $0.5<d>0.75$ \\
IV & Moderately Severe & $0.25<d>0.5$ \\
V & Severe & $d>0.25$ \\
VI & Total Paralysis & $d=0$
\end{tabular}

${ }^{*} d=$ distance between location of point on neutral expression image and that in test image

AAM also has been used in previous study by Luise and Joachim [12], where the facial paralysis severity was automatically predicted. The proposed framework consists of three stages; half sided AAM was trained on images of healthy persons with both neutral expressions and performing exercises. Then, this trained AAM was fitted in patient images using multivariate linear regression. The Euclidean distance between the landmarks and their neighbors were calculated in order to describe the movement of these landmarks. The distances between the landmarks should be larger by comparing to the distances between two healthy hemispheres if the paralyzed half of the face is not able to do the exercise precisely. This information was combined with the labeled facial paralysis indices (HBGS), and then was used to train a Random Decision Forest classifier. The prediction rates of indices were obtained by performing a 5-fold cross-validation and a parameter analysis in finding the best combination of parameters for an optimal classification. To train and predict the HBGS index, 165 different dates of experiment were used and the predicted indices are grouped in the middle as illustrated in Table 4.

As many other works, Amira et al [13] have also modified HBGS in order to adapt with their proposed work. This modified HBGS is sum of two scores; eyebrows movement score and mouth movement score. The total maximum score is $8 / 8$ as illustrated in Table 5 where it reflects a healthy subject with normal facial function. The eyebrow movement score was calculated by measuring upwards movement of the eyebrow (ME) while the mouth movement score was calculated by measuring the outwards movement of mouth corner (MC). For each $0.25 \mathrm{~cm}$ movement, a score of 1 was assigned with the maximum of $1 \mathrm{~cm}$ movement for each portion.

Table 4. Distribution of all House-Brackmann indices that were used for prediction during crossvalidation (predictions in row and ground-truth in columns)

\begin{tabular}{ccccccc}
\hline I & I & II & III & IV & V & VI \\
\hline I & 0 & 0 & 0 & 0 & 0 & 0 \\
II & 0 & 0 & 2 & 2 & 0 & 0 \\
III & 3 & 18 & 33 & 27 & 20 & 3 \\
IV & 0 & 21 & 17 & 15 & 3 & 0 \\
V & 0 & 0 & 1 & 0 & 0 & 0 \\
VI & 0 & 0 & 0 & 0 & 0 & 0 \\
Dist: & 3 & 39 & 53 & 44 & 23 & 3 \\
\hline
\end{tabular}

Table 5. Presentation of modified HBGS

\begin{tabular}{cccc}
\hline Grade & Score & Function $\%$ & Estimated Function \\
\hline I & $8 / 8$ & 100 & 100 \\
II & $7 / 8$ & $76-99$ & 80 \\
III & $5 / 8-6 / 8$ & $51-75$ & 60 \\
IV & $3 / 8-4 / 8$ & $26-50$ & 40 \\
V & $1 / 8-2 / 8$ & $1-25$ & 20 \\
VI & $0 / 8$ & 0 & 0 \\
\hline
\end{tabular}


Shu He et al [14] have presented an approach where optical flow was calculated in identifying the direction and amount of movement between the image sequences. This optical flow was computed using their proposed algorithm to measure the symmetry of facial movements between both sides of face. Then, these results were combined with the total pixel intensity changes and an illumination factor in specific regions before fed into classifiers to estimate the degree of movement by using normal side as base line. In order to get the HB score, these regional results were fed into another classifier. The inputs which were involved and correlated with HBGS are ratio of total pixel change between normal and dysfunction side of face, the illumination factor, $\operatorname{Sym}_{\mathrm{y}}$ (symmetry relative to vertical component of total amount of displacements from rest to maximum of movement) and $\mathrm{Sym}_{\mathrm{r}}$ (symmetry relative to the strength of total amount of displacements from rest to maximum of movement).

The optical flow estimation also has been used in work proposed by Wang and Qi [15]. This approach has used $P_{\text {face, }}$ which is stem from $D_{\text {face }}$ (density of difference face) [16] to measure the asymmetry between sides of face. From the experiments, the value of $D_{\text {hy }}$ (face is at rest) was nearly does not change, $P_{\text {face }}$ was closed to zero and $P_{\text {face }}$ became smoothed zero surface for normal subjects. In other hands, in patient cases, $D_{\text {hy }}$ value lose the stability and $P_{\text {face }}$ surface's altitude were increased at first then reached maximum at apex before shrinked to zero at the ending. The final results were based on the threshold of $P_{\text {face }}$ which was defined as 4.0. The test subject was claimed as patient with facial paralysis if the value of $P_{\text {face }}$ was larger than 4.0. As in Table 6, the experiments results showed the HB scores based on total $P_{\text {face }}$ calculated for each subject.

Table 6. Correlation of Total $P_{\text {face }}$ with HBGS

\begin{tabular}{cccc}
\hline Subject & Age & Total $P_{\text {face }}$ & HBGS \\
\hline 1 & 38 & 1.5624 & I \\
2 & 23 & 2.2463 & I \\
3 & 18 & 1.0274 & I \\
4 & 34 & 1.0012 & I \\
5 & 54 & 0.9264 & I \\
6 & 45 & 6.3754 & II \\
7 & 36 & 7.4026 & II \\
8 & 60 & 8.6316 & II \\
9 & 25 & 11.625 & II \\
10 & 64 & 18.864 & III \\
\hline
\end{tabular}

Recent work by Barbosa et al [17], have also utilized HBGS in order to get the severity level for each investigated facial region; forehead, eye and mouth region. A hybrid classifier which combined a rule-based expert system and machine learning is proposed. The process of leveling the severity of paralysis consists of two rules; Rule 1 and Rule 2. The algorithm was continued to move if Rule 1 is satisfied, then run a test if Rule 2 is also satisfied. Apart from, machine learning task was performed. For example, if condition $\mathrm{f} 10<0.95$ and $\mathrm{f} 8<0.95$ happened in Rule 1 as shown in Table 7, then the subject was most likely diagnosed with facial paralysis, and may continue to Rule 2 where the facial palsy classification was conducted; otherwise it performed a machine learning task. The classifier may exit from the whole process and the subject was classified as healthy if the classifier returns 0. By using HBGS in determining the degree of severity of paralysis, they have tested each region grades (e.g. if mouthGrade $=2$, foreheadGrade $=2$ and eyeGrade $=2$, using to HBGS, the overall grade is 3 which is moderate).

In the same year, Syahirah and Kenneth [18] have proposed an individual score chart as shown in Table 8 for assigning a score to each facial region. The score was assigned based on the percentage of difference value of area measurement. 
Table 7. List of features

\begin{tabular}{lc}
\hline Asymmetrical features & Parameters \\
\hline Iris area while lifting eyebrows with both eyes directed upward & $\mathrm{f} 1$ \\
Rate of movement from rest to lifting eyebrows (use distance & $\mathrm{f} 2$ \\
between supra orbital (SO) and upper part of occluded iris) & \\
Rate of movement from rest to lifting eyebrows (use distance & $\mathrm{f} 3$ \\
between SO and infra orbital (IO) ) & $\mathrm{f} 4$ \\
Distance between SO and IO while lifting eyebrows & $\mathrm{f5}$ \\
Distance between SO and upper boundary of occluded iris & $\mathrm{f6}$ \\
while raising eyebrows with both eyes looking upward & $\mathrm{f} 7$ \\
Distance between SO and IO while closing both eyes & $\mathrm{f} 8$ \\
Iris area while showing teeth or smiling & $\mathrm{f} 9$ \\
Distance between IO and mouth angle while smiling & $\mathrm{f} 10$ \\
Iris area while screwing nose & \\
Mean ratio of features 1-9 &
\end{tabular}

Table 8. Individual score chart

\begin{tabular}{cc}
\hline$\% \Delta \mathrm{A}$ & Score assigned \\
\hline$<1$ & 0 \\
$1-5$ & 1 \\
$6-10$ & 2 \\
$11-15$ & 3 \\
$16-20$ & 4 \\
$21-25$ & 5 \\
26 and above & 6 \\
\hline
\end{tabular}

Table 9. Grading of paralysis based on the total score

\begin{tabular}{ccc}
\hline House-Brackmann grade & Descriptions & Total Score \\
\hline I & Normal & $22-36$ \\
II & Slight Dysfunction & $11-21$ \\
III & Moderate Dysfunction & $7-10$ \\
IV & Moderate Severe Dysfunction & $4-6$ \\
V & Severe Dysfunction & $1-3$ \\
VI & Total Paralysis & 0 \\
\hline
\end{tabular}

Then, another table was constructed as illustrated in Table 9 where the HouseBrackmann grade or level was assigned based on the total score from three regions; brow, eye and mouth regions. Based on table, a total score in range of 0 to 21 has indicated that the subject is a patient with a level of severity of paralysis, whereas a total score in the range of 22 to 36 has indicated that the subject exhibits normal facial nerve function. If the subjects was assessed as abnormal, then based on individual score chart, the scores will be obtained for the right and left side of face. Hence, the paralysis side of face will be known after the individual score was assigned.

\subsection{Relationship between Objective Assessment Methods}

From all of these objective methods which are correlated with House-Brackmann (HB), it has been found that there are a few relationships between these reviewed methods. Obviously, they have used digital images in analyzing the changes on certain areas of face. The differences in ratio between the right and left sides of face have been evaluated based on facial movement and also in rest condition. Usually, patients have to perform a particular facial movement which started from a neutral face expression and then gradually shifted to the maximal movement before they return to neutral expression again. 


\section{Conclusion}

A brief overview on eight articles of previous objective facial nerve assessments which have correlated their works with House-Brackmann Grading System (HBGS) has been presented in this article. From eight articles, it comes to a conclusion that the main important facial parts in human face is consists of three main regions which are forehead, eye and mouth region. Besides, the review showed that even though the HBGS has its own disadvantages, this system is still being used and become as ground truth in proposing a facial nerve assessment method. This is due to the global interests in such of simple and fast evaluation system like HBGS. The objective facial nerve assessment methods based on HBGS should be extended to a high level where the system will automatically assessed the paralysis patient and monitor the improvement during rehabilitation since HBGS is used worldwide. Many extensive works should be done and publicly available database should be encouraged to increase the work done in this area

\section{Acknowledgement} (FRGS).

This material is based upon work funded by the Fundamental Research Grant Scheme

\section{References}

[1] Brach JS, VanSwearingen JM. Not all facial paralysis is Bell's palsy: a case report. Arch Phys Med Rehabil. 1999; 80(7): 857-859.

[2] Jackson CG and Doersten PG. The facial nerve. Current trends in diagnosis: treatment and rehabilitation. Med Clin North Am. 1999; 83(1): 179-195.

[3] Scheller K, Scheller C. Nimodipine promotes regeneration of peripheral facial nerve function after traumatic injury following maxillofacial surgery: an off label pilot-study. J Craniomaxillofac Surg. 2012; 40(5): 427-434.

[4] House JW. Facial nerve grading systems. Laryngoscope. 1983; 93(8): 1056-69.

[5] House JW and Brackmann DE. Facial nerve grading system. Otolaryngol Head Neck Surg. 1985; 93(2): 146-7.

[6] Samsudin WSW, Sundaraj K. Evaluation and Grading Systems of Facial Paralysis for Facial Rehabilitation. J Phys Ther Sci. 2013; 25(4): 515-19.

[7] Fattah AY, Gurusinghe D, Gavilan J et al. Reply: facial nerve grading scales: systematic review of the literature and suggestion for uniformity. Plastic \& Reconstructive Surgery. 2015; 135(5): 930-31.

[8] Vrabec JT, Backous DD, Djalilian HR et al. Facial Nerve Grading System 2.0. Otolarngol Head Neck Surg. 2009; 140(4): 445-50.

[9] Yen TL, Driscoll CL, Lalwani AK. Significance of House-Brackmann facial nerve grading global score in the setting of differential facial nerve function. Otol Neurotol. 2003; 24(1): 118-22.

[10] Wang T, Zhang S, Dong $\mathrm{J}$ et al. Automatic Evaluation of the Degree of Facial Nerve Paralysis. Multimed Tools Apl. 2015; 75(19): 11893-11908.

[11] Delannoy JR, Ward TE. A preliminary investigation into the use of Machine Vision Techniques for Automating Facial Rehabilitation Therapy. 2010 ISSC Proc IET Irish. Ireland. 2010: 228-232.

[12] Modersohn L, Denzler J. Facial paresis index prediction by exploting active appearance models for compact discriminative features. International Conference on Computer Vision Theory and Applications (VISAPP). 2016: 271-278.

[13] Gaber A, Faher MF, Wahed MA. Automated grading of facial paralysis using the Kinect v2: A proof concept study. International Conference on Virtual Rehabilitation Proceedings (ICVR). Egypt. 2016: 258-264.

[14] He S, Soraghan JJ, o'Reilly B. Biomedical Image Sequence Analysis with Application to Automatic Quantitative Assessment of Facial Paralysis. EURASIP Journal on Image and Video Processing. 2007.

[15] Wang S, Qi F. Compute Aided Diagnosis of Facial Paralysis Based on Pface. IEEE Engineering in Medicine and Biology $27^{\text {th }}$ Annual Conference. 2005: 4353-4356.

[16] Liu Y, Mitra S. Facial Asymmetry Quantification for Expression Invariant Human Identification. Computer Vision and Image Understanding Journal. 2003; 91(1-2): 138-159.

[17] Barbosa J, Lee K, Lee $S$ et. al. Efficient quantitative assessment of facial paralysis using iris segmentation and active contour -based key points detection with hybrid classifier. BMC Medical Imaging. 2016; 16: 23.

[18] Samsudin WSW, Sundaraj K, Ahmad A et al. Initial assessment of facial nerve paralysis based on motion analysis using an optical flow method. Technol Health Care. 2016; 24(2): 287-99. 